\title{
On the inverse hoop conjecture of Hod
}

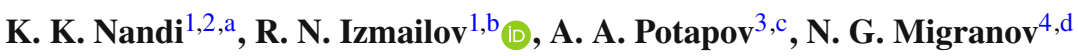 \\ ${ }^{1}$ Zel'dovich International Center for Astrophysics, M. Akmullah Bashkir State Pedagogical University, 3A, October Revolution Street, \\ Ufa, RB 450008, Russia \\ ${ }^{2}$ High Energy Cosmic Ray Research Center, University of North Bengal, Darjeeling, WB 734 013, India \\ ${ }^{3}$ Department of Physics and Astronomy, Bashkir State University, 47A, Lenin Street, Sterlitamak, RB 453103, Russia \\ ${ }^{4}$ Department of Medical Physics and Informatics, Bashkir State Medical University, 3, Lenina Street, Ufa, RB 450008, Russia
}

Received: 23 September 2021 / Accepted: 30 October 2021 / Published online: 13 November 2021

(c) The Author(s) 2021

\begin{abstract}
Recently, Hod has shown that Thorne's hoop conjecture $\left(\frac{C(R)}{4 \pi M(r \leq R)} \leq 1 \Rightarrow\right.$ horizon) is violated by stationary black holes and so he proposed a new inverse hoop conjecture characterizing such black holes (that is, horizon $\left.\Rightarrow \mathcal{H}=\frac{\pi \mathcal{A}}{\mathcal{C}_{\text {eq }}^{2}} \leq 1\right)$. In this paper, it is exemplified that stationary hairy black holes, endowed with Lorentz symmetry violating Bumblebee vector field related to quantum gravity and dilaton field of string theory, also respect the inverse conjecture. It is shown that stationary hairy singularity, recently derived by Bogush and Galt'sov, does not respect the conjecture thereby protecting it. However, curiously, there are two horizonless stationary wormholes that can also respect the conjecture. Thus one may also state that throat $\Rightarrow \mathcal{H} \leq 1$, suggesting that the inverse conjecture may be a necessary but not sufficient proposition.
\end{abstract}

\section{Introduction}

Thorne's hoop conjecture is of fundamental importance in black hole physics and has since been a topic of great interest in the literature. However, Hod [1] has shown that the conjecture is intriguingly violated for spinning black holes and a unified version of the conjecture applicable to black holes and charged horizonless compact objects is thus unavailable. With this in mind, Hod [2] has introduced a new geometrical proposal that stationary black holes are characterized by an inverse hoop conjecture $\mathcal{A} \leq \frac{\mathcal{C}^{2}}{\pi}$, where $\mathcal{A}$ and $\mathcal{C}$ are respectively the black hole surface area and the circumference length of the smallest ring that can engulf the black hole

\footnotetext{
a e-mail: kamalnandi1952@ rediffmail.com

be-mail: izmailov.ramil@gmail.com (corresponding author)

c e-mail: a.a.potapov@strbsu.ru

de-mail: ufangm@yahoo.co.uk
}

horizon in every direction. This area-circumference inequality has been exemplified by Hod [2] for the generic KerrNewman-(anti)-de Sitter black holes:

$\Delta_{r}\left(r_{+}\right)=0$,

$\mathcal{H}(M, Q, a, \Lambda)=\frac{\pi \mathcal{A}}{\mathcal{C}_{\mathrm{eq}}^{2}}=\frac{\left(1+\frac{1}{3} \Lambda a^{2}\right) r_{+}^{2}}{r_{+}^{2}+a^{2}} \leq 1$,

where $\Delta_{r}(r)=r^{2}-2 M r+a^{2}+Q^{2}-\frac{1}{3} \Delta r^{2}\left(r^{2}+a^{2}\right)+Q^{2}$, $r=r_{+}$is the horizon radial coordinate, $\mathcal{C}_{\text {eq }}$ is the equatorial circumference length of horizon, $Q$ is the electric charge, $a$ is the spin, $\Lambda$ is the cosmological constant (It would be only fair to call $\mathcal{H}$ the Hod function, a prototype of which for static black holes was already defined and denoted by $H$ in [3]). The inequality (2) embodying the inverse conjecture is consistent also with a time-dependent (oscillating) equatorial and polar circumferences of non-stationary black hole horizons [4].

The purpose of this paper is to explicitly demonstrate that Hod's inverse conjecture is respected also by spinning hairy black holes such as the Lorentz symmetry violating Bumblebee field related to quantum gravity and dilaton field of string theory, while singularity with scalar hair does not respect it thereby protecting the conjecture. However, the conjectured inequality $\mathcal{H} \leq 1$ describing stationary black hole horizon cannot exclude stationary uncharged horizonless wormholes suggesting that the inverse conjecture may be necessary but not a sufficient condition.

\section{Bumblebee gravity spinning black hole}

The spinning Kerr-like black hole [5] metric in the Bumblebee gravity in Boyer-Lindquist coordinates is

$d \tau^{2}=g_{t t} d t^{2}+g_{r r} d r^{2}+g_{\theta \theta} d \theta^{2}+g_{\varphi \varphi} d \varphi^{2}+2 g_{t \varphi} d t d \varphi$, 
where $\ell \geq 0$ is the Lorentz symmetry violating parameter defined by $\lambda=\sqrt{1+\ell}$ and

$$
\begin{aligned}
g_{t t} & =-\left(1-\frac{2 M r}{\Sigma}\right), \\
g_{r r} & =\frac{\Sigma}{\Delta}, \\
g_{\theta \theta} & =\Sigma, \\
g_{\varphi \varphi} & =\frac{\Xi}{\Sigma} \sin ^{2} \theta, \\
g_{t \varphi} & =\frac{2 a M r \lambda}{\Sigma} \sin ^{2} \theta, \\
\Sigma & =r^{2}+\lambda^{2} a^{2} \cos ^{2} \theta, \\
\Delta & =\frac{r^{2}-2 M r}{\lambda^{2}}+a^{2}, \lambda \neq 0 \\
\Xi & =\left(r^{2}+\lambda^{2} a^{2}\right)^{2}-\Delta \lambda^{4} a^{2} \sin ^{2} \theta .
\end{aligned}
$$

Following the method as outlined in [2], we obtain, using $\Delta\left(r_{+}\right)=0$,

$$
\begin{aligned}
\mathcal{A} & =4 \pi\left(r_{+}^{2}+\lambda^{2} a^{2}\right), \\
\mathcal{C}_{\mathrm{eq}} & =2 \pi\left(\frac{r_{+}^{2}+\lambda^{2} a^{2}}{r_{+}}\right), \\
\mathcal{H} & =\frac{\pi \mathcal{A}}{\mathcal{C}_{\mathrm{eq}}^{2}}=\frac{r_{+}^{2}}{r_{+}^{2}+\lambda^{2} a^{2}} \leq 1,
\end{aligned}
$$

which is what we wanted to explicitly demonstrate. Equality holds when $a=0$.

\section{Kerr-Sen stringy spinning black hole}

This black hole of heterotic string theory [6-9] in BoyerLindquist coordinates in the Einstein frame is given by the metric components with the definitions

$$
\begin{aligned}
& \Sigma=r^{2}+a^{2} \cos ^{2} \theta+2 r \xi \\
& \Delta=r^{2}-2 m r+a^{2}, \\
& \Xi=\left(r^{2}+a^{2} \cos ^{2} \theta+2 r \xi\right)^{2}-\Delta a^{2} \sin ^{2} \theta,
\end{aligned}
$$

where $\xi$ is the string parameter defined by

$\xi=m \sinh ^{2} \alpha$,

and the mass $M$, dilaton charge $Q$ and spin $J$ are parametrized as

$$
\begin{aligned}
M & =\frac{m}{2}(1+\cosh 2 \alpha), \quad Q=\frac{m}{\sqrt{2}} \sinh 2 \alpha, \\
J & =\frac{a m}{2}(1+\cosh 2 \alpha) .
\end{aligned}
$$

At $\xi=0$, one recovers the Kerr solution. Following the method as outlined in [2], we obtain, using $\Delta\left(r_{+}\right)=0$,

$$
\begin{gathered}
\mathcal{A}=4 \pi\left(r_{+}^{2}+a^{2}+2 r_{+} \xi\right), \\
\mathcal{C}_{\text {eq }}=2 \pi \frac{r_{+}^{2}+a^{2}+2 r_{+} \xi}{\sqrt{r_{+}^{2}+2 r_{+} \xi}}
\end{gathered}
$$

so that

$\mathcal{H}=\frac{r_{+}^{2}+2 r_{+} \xi}{r_{+}^{2}+a^{2}+2 r_{+} \xi} \leq 1$.

Once again, equality holds only in the spinless case, $a=0$.

\section{Bogush-Galt'sov spinning singularity}

A recent example of exact spinning singular solution belonging to Einstein's gravity of minimally coupled scalar field $\phi$ is given by Bogush and Galt'sov [10]. The general form of the metric is given by

$d \tau^{2}=-f\left(d t-\omega_{i} d x^{i}\right)^{2}+f^{-1} h_{i j} d x^{i} d x^{j}$,

where the function $f$, the one-form $\omega_{i}$ and the 3-metric $h_{i j}$ are functions of space coordinates $x^{i}(i=1,2,3)$. The relevant functions are

$$
\begin{aligned}
f(r, \theta) & =\frac{\Delta-a^{2} \sin ^{2} \theta}{r^{2}+a^{2} \cos ^{2} \theta}, \\
\omega(r, \theta) & =-\frac{2 a M r \sin ^{2} \theta}{\Delta-a^{2} \sin ^{2} \theta}, \\
h_{i j} d x^{i} d x^{j} & =H(r, \theta)\left(d r^{2}+\Delta d \theta^{2}\right)+\Delta \sin ^{2} \theta d \varphi^{2}, \\
H(r, \theta) & =\frac{\Delta-a^{2} \sin ^{2} \theta}{\Delta}\left(1+\frac{b^{2} \sin ^{2} \theta}{\Delta}\right)^{-\frac{\Sigma^{2}}{b^{2}}}, \\
\Delta(r) & =(r-M)^{2}-b^{2}, \\
\phi & =\phi_{\infty}+\frac{\Sigma}{2 b} \log \frac{r-M+b}{r-M-b}, \\
b & =\sqrt{M^{2}-a^{2}} \Rightarrow b<M .
\end{aligned}
$$

Note that $\Sigma=0$ leads to the Kerr black hole. The Ricci scalar is

$R=\frac{2 \Sigma^{2}}{\Delta\left(r^{2}+a^{2} \cos ^{2} \theta\right)}\left(1+\frac{b^{2} \sin ^{2} \theta}{\Delta}\right)^{\frac{\Sigma^{2}}{b^{2}}}$,

which diverges on the "horizon" $\Delta\left(r_{\mathrm{s}}\right)=0$ (inverted commas by the authors of [10]). The singular radius is

$r_{\mathrm{s}}=M+b, b^{2}>0$. 
In the extremal limit $b^{2}=M^{2}-a^{2} \rightarrow 0$,

$$
\begin{aligned}
H & =\frac{\Delta-a^{2} \sin ^{2} \theta}{\Delta} \exp \left(-\frac{\Sigma^{2} \sin ^{2} \theta}{\Delta}\right), \\
\phi & =\phi_{\infty}+\frac{\Sigma}{\sqrt{\Delta}} .
\end{aligned}
$$

The Bogush-Galt'sov singular solution satisfies all energy conditions since the scalar field couples to gravity with correct sign. Let us find the equatorial circumference length of horizon $\mathcal{C}_{\text {eq }}$ under the conditions $d t=0, d r=0$ yielding from the metric (23-30) the surface metric

$d S^{2}=f^{-1} H \Delta d \theta^{2}+\left(f^{-1} \Delta \sin ^{2} \theta-f \omega^{2}\right) d \varphi^{2}$.

On the equatorial plane, $\theta=\pi / 2$, so the total length encircling the singular equator $\Delta\left(r_{\mathrm{s}}\right)=0$ with $d \varphi \rightarrow 2 \pi$ is given by

$$
\begin{aligned}
\mathcal{C}_{\text {eq }} & =2 \pi \sqrt{-f \omega^{2}} \\
& =2 \pi .2 M
\end{aligned}
$$

which is surprisingly independent of $r_{\mathrm{s}}$ but the area of the singular surface is

$$
\begin{aligned}
\mathcal{A}= & 2 \pi \int_{0}^{\pi} \sqrt{p q} d \theta \\
p= & f^{-1} H \Delta \\
q= & f^{-1} \Delta \sin ^{2} \theta-f \omega^{2} \\
\sqrt{p q}= & {\left[( \Delta - a ^ { 2 } \operatorname { s i n } ^ { 2 } \theta ) \Delta \left\{\left(r^{2}+a^{2} \cos ^{2} \theta\right)^{2}\right.\right.} \\
& \left.\left.-4 a^{2} r^{2} M^{2} \sin ^{2} \theta\right\}\left(1+\frac{b^{2} \sin ^{2} \theta}{\Delta}\right)^{-\frac{\Sigma^{2}}{b^{2}}}\right]^{-1 / 2} .
\end{aligned}
$$

Noting that, when $\Delta\left(r_{s}\right) \rightarrow 0,\left(1+\frac{b^{2} \sin ^{2} \theta}{\Delta}\right)^{-\frac{\Sigma^{2}}{b^{2}}} \rightarrow 0$, we have $\sqrt{p q} \rightarrow \infty$ so that

$\mathcal{A} \rightarrow \infty$.

Thus, it follows that

$\mathcal{H}=\frac{\pi \mathcal{A}}{\mathcal{C}_{\text {eq }}^{2}}=\infty$

which is consistent with the singular nature of the solution. Thus the conjecture applicable for black holes is preserved. We next consider two examples of horizonless compact objects known as wormholes.

\section{Damour-Solodukhin stationary wormhole}

The static Damour-Solodukhin wormhole [11] has been generalized to a stationary wormhole by Bueno et al [12] having the metric of the same form as (3-11) but with only a redefined $\Delta \rightarrow \widehat{\Delta}$ given by

$$
\begin{aligned}
\Sigma & =r^{2}+a^{2} \cos ^{2} \theta, \\
\widehat{\Delta} & =r^{2}-2 M\left(1+\delta^{2}\right) r+a^{2}, \delta \neq 0 \\
g_{\varphi \varphi} & =\left[\left(r^{2}+a^{2}\right)+\frac{2 M r a^{2} \sin ^{2} \theta}{\Sigma}\right] \sin ^{2} \theta,
\end{aligned}
$$

where $\delta$ is the parameter representing deviation from Kerr black hole. Physical and geometrical properties have been well studied. For instance, Bueno et al [12] studied quasinormal modes of gravitational waves and Karimov et al [13] studied its accretion signatures. These studies compared the wormhole signatures with those of Kerr black holes that could be of interest in the experimental search for wormholes.

The throat (not a horizon [12]) appears at

$$
\widehat{\Delta}=0 \Rightarrow r_{\text {th }}=\left(1+\delta^{2}\right) M+\sqrt{M^{2}\left(1+\delta^{2}\right)^{2}-a^{2}} .
$$

The angular part of the metric is that of the Kerr and hence it directly follows that

$\mathcal{H}=\frac{\pi \mathcal{A}}{\mathcal{C}_{\text {eq }}^{2}} \leq 1$.

The inverse conjecture is obviously well satisfied.

\section{Teo stationary wormhole}

This is also a well discussed spinning wormhole [14] having a general form of the metric

$$
\begin{aligned}
d \tau^{2}= & -N^{2} d t^{2}+\frac{d r^{2}}{1-\frac{b(r)}{r}} \\
& +r^{2} K^{2}\left[d \theta^{2}+\sin ^{2} \theta(d \varphi-\omega d t)^{2}\right],
\end{aligned}
$$

where the arbitrary functions $N, K$ and $\omega$ depend only on $r$ and $\theta$. The topology of the wormhole, distinct from that of a black hole, has been discussed in detail by the author himself [14] and several others (see, e.g., [15]) and hence omitted here. The function $b$, called the shape function, has to be independent of $\theta$ to avoid curvature singularity, that is, $\partial_{\theta} b(r, \theta)=0$. The metric represents two identical regions, interior and exterior, that are joined at the throat $r=b\left(r_{\text {th }}\right)=r_{\text {th }}$. The redshift function $N(r, \theta)$ should be horizonless for traversability as required in the standard Morris-Thorne wormhole, i.e., $N$ should be finite and nonzero, so that no curvature singularities and event horizons occur. But if we do not require physical traversability, there can be horizons such that $N(r, \theta)$ can be zero at some chosen radius but $\mathcal{H}$ is independent of $N(r, \theta)$. Remarkably, it has been shown in [14] that for some choices of $N, K$ and $\omega$, the wormhole though it has no horizon can nonetheless have ergoregions around the throat from where energy could be extracted by the Penrose process. 
The circumferential length of the throat and the area of the throat surface are respectively $\left(d t=0, r=r_{\text {th }}, \theta=\pi / 2\right.$, $\varphi \rightarrow 2 \pi)$ :

$$
\begin{aligned}
\mathcal{C}_{\mathrm{eq}} & =2 \pi r_{\mathrm{th}} K \\
\mathcal{A} & =4 \pi r_{\mathrm{th}}^{2} K^{2}
\end{aligned}
$$

yielding

$\mathcal{H}=\frac{\pi \mathcal{A}}{\mathcal{C}_{\text {eq }}^{2}}=1$.

Here the conjecture is marginally satisfied.

\section{Remarks}

The above results indicate that the unified inverse hoop conjecture (horizon $\Rightarrow \mathcal{H} \leq 1$ ) due to Hod has come to stay for stationary black holes having the same status as that of Thorne's hoop conjecture for static black holes. However, unlike the former which involves mass, the latter is defined purely geometrically depending only on the angular part of a given metric. For instance, $\mathcal{H}=1$ holds trivially on any spherical surface even in flat spacetime. In principle, however, any metric could be regarded as a black hole solution of general relativity provided we are ready to accept physically uninteresting forms of stress tensor and such spacetimes may be tailored to violate the relation $(\mathcal{H} \leq 1)$, a concern already noted in [2]. Singular solutions may appear even with well known stress tensors such as the Bogush-Galt'sov solution for minimally coupled scalar field, which however protects the conjecture by violating it.

Two curious examples of stationary horizonless wormholes that have been well studied since their very inception. It was pointed out above that they can also respect the conjecture. This suggests that one may regard $\mathcal{H} \leq 1$ as a necessary but not a sufficient condition since the conjecture allows wormholes (uncharged horizonless compact objects) for which one may state that: throat $\Rightarrow \mathcal{H}=\frac{\pi \mathcal{A}}{\mathcal{C}_{\mathrm{eq}}^{2}} \leq 1$. However, the main purpose of this paper was to point out the efficacy of the conjecture going beyond Kerr-Newman-de Sitter black holes by extending it to the regime of stationary hairy black holes.
Data Availability Statement This manuscript has no associated data or the data will not be deposited. [Authors' comment: This is a theoretical study and no experimental data has been listed.]

Open Access This article is licensed under a Creative Commons Attribution 4.0 International License, which permits use, sharing, adaptation, distribution and reproduction in any medium or format, as long as you give appropriate credit to the original author(s) and the source, provide a link to the Creative Commons licence, and indicate if changes were made. The images or other third party material in this article are included in the article's Creative Commons licence, unless indicated otherwise in a credit line to the material. If material is not included in the article's Creative Commons licence and your intended use is not permitted by statutory regulation or exceeds the permitted use, you will need to obtain permission directly from the copyright holder. To view a copy of this licence, visit http://creativecomm ons.org/licenses/by/4.0/.

Funded by SCOAP ${ }^{3}$.

\section{References}

1. S. Hod, Eur. Phys. J. C 80, 982 (2020)

2. S. Hod, Eur. Phys. J. C 80, 1148 (2020)

3. K.K. Nandi, R.N. Izmailov, G.M. Garipova, R.R. Volotskova, A.A. Potapov, Phys. Lett. B 809, 135734 (2020)

4. W.E. East, Phys. Rev. Lett. 122, 231103 (2019)

5. C. Ding, C. Liu, R. Casana, A. Cavalcante, Eur. Phys. J. C 80, 178 (2020)

6. R.P. Kerr, Phys. Rev. Lett. 11, 237 (1963)

7. A. Sen, Phys. Rev. Lett. 69, 1006 (1992)

8. A. Bhadra, T.B. Nayak, K.K. Nandi, Phys. Lett. A 295, 1 (2002)

9. R.N. Izmailov, RKh. Karimov, A.A. Potapov, K.K. Nandi, Ann. Phys. 413, 168069 (2020)

10. I. Bogush, D. Galt'sov, Phys. Rev. D 102, 124006 (2020)

11. T. Damour, S.N. Solodukhin, Phys. Rev. D 76, 024016 (2007)

12. P. Bueno, P.A. Cano, F. Goelen, T. Hertog, B. Vercnocke, Phys. Rev. D 97, 024040 (2018)

13. RKh. Karimov, R.N. Izmailov, K.K. Nandi, Eur. Phys. J. C 79, 952 (2019)

14. E. Teo, Phys. Rev. D 58, 024014 (1998)

15. P.G. Nedkova, V.K. Tinchev, S.S. Yazadjiev, Phys. Rev. D 88, 124019 (2013) 